\title{
SIMULTANEOUS BILATERAL RODENT ULCER OF CORNEA, CURED BY COMBINED CURETTING, THERMOCAUTERY AND MASSIVE CYANIDE SUBCONJUNCTIVAL INJECTION
}

\author{
BY
}

\author{
E. L. JONES, M.D. \\ CUMBERLAND, MARYLAND, U.S.A.
}

DUE to the rarity and obstinate resistance to treatment of the. disease as above titled, it seems a suitable subject to bring before the profession to elicit expression by those of larger experience both as to diagnostic limitations, and means for its cure. From what I have been able to read, all commentators agree as to its origin at the corneal margin, and its slowly progressive destruction of the corneal surface, leaving opaque cornea when it heals, and seldom, if ever, penetrating the entire cornea. Yet some speak in terms as though marginal ulcer, ulcus serpens, etc., are to be confused with rodent ulcer or Mooren's ulcer, as it has been more frequently called since this oculist brought it into conspicuous notice. Reading some high authorities one might think all that was necessary to cure it was a single use of actual cautery; but reading case reports shows how certain ulcers have gone ruthlessly on in spite of many cauterizations, as well as other orthodox methods, to a long drawn-out and tragic destruction of one, and sometimes later on, the other eye of its victim.

The first exhaustive description of rodent ulcer, with compiled literature on the subject, to come to my observation, was the paper by the late Dr. Randolph presented before the eve section of the Amer. Med. Assoc., and published in the transactions for 1909, p. 308. Even after reading that, it was lost to memory until a year or two later. My first case was encountered in the person of an elderly Italian man. It was only after failure of expected benefit from usual treatments that the Randolph paper was thought of. The patient in the meantime decided to return to his native land, so that further efforts were ended. He was told to go to an oculist in the nearest city to his home, which happened to be Turin. In the more than 20 years since then, a small number of what were judged to be rodent ulcers in one eye only have been successfully treated by the measures hereinafter to be described. Dr. Bedell has a report, with later literature, of two cases, both ending in loss of eyes, in the Amer. Jl. of Ophthal. for April, 1933, p. 289. The stereoscopic picture he exhibits is exactly like the case later to be presented as to the size, location and shape of ulceration. A recent paper by Dr. Sandford Gifford, reporting 
three cured cases in the Arch. of Ophthal. for December, 1933, p. 800 , gives a much more roseate prognosis than heretofore justified.

In the Arch. of Ophthal. for May, 1934, p. 832, a case is reported by F.W. Dean, of a patient losing one eye from Mooren's ulcer in spite of all the then taught methods of treatment. A short while after the remaining eye became affected, and was progressively growing worse in spite of everything, when the idea of attacking the engorged conjunctival area by cautery and curetting, and then covering the affected area by a sliding conjunctival flap, proved the key to a successful cure.

The suddenness with which the condition can arise, as demonstrated by my patient's second eye, inclines me to believe that the origin is neuropathic. Likewise the corneal anaesthesia, persisting a month after healing does the same. Time had not allowed the extensive undermining of the sclera depicted in the Randolph illustration, but there were heavy, thick vascular pads extending on to the adjacent sclera, which bled profusely when they, along with the ulcerated cornea, were vigorously curetted.

Mrs. M., aged 60 years, a large, robust country woman, presented herself on May 25, 1933, complaining of a hot, burning, and slightly painful feeling of only a few days duration in her right eye. The conjunctival vessels were everywhere engorged, and the upper cornea near the limbus resembled a heavy arcus senilis. This did not stain green with fluorescein, but a dirty yellow. The scleral area adjacent was, as before mentioned, a thick vascular pad. Neither then nor at any subsequent time, was there evidence of iritis, as measured by appearance or tenderness to touch. The supposed nature of the disease was explained to her, with the necessity of vigorous measures of treatment required. But it was decided to try one simple treatment of rubbing the ulcer with cotton-wound tooth-pick moistened with carbolic acid, and argyrol eye drops. The patient was permitted to go to her home in the country for two days with the understanding that active measures were to be used on her return, if the conditions were no better. She was taking occasional treatments with slight restrictions on diet, from a general physician. Inquiry of him elicited the information that all he could find amiss was a slight rise in blood pressure, and occasionally a little albumin in the urine. On her return on May 27, the ulceration was extending. After cocainizing the eye, novocaine was injected subconjunctivally for anaesthesia. With a small mastoid curette, the ulcer and adjacent sclera were forcibly curetted, with profuse bleeding resulting, until a trough almost the entire thickness of cornea about three millimetres wide at the middle, and about one-fourth of the circumference was made. This trough, in its entite area, was seared with a heavy paddle-shaped 
post-thermocautery, and a massive subconjunctival injection of cyanide of mercury ( 2 c.c. of $1 / 1,500$ sol.) immediately given. After giving this injection, the eye is always left unbandaged for half an hour or so, to let the patients or their relations see that the enormous swelling of the lids and conjunctiva which has been expected and desired has taken place. The eye is then bandaged, and the pads changed daily as long as the profuse serous discharge continues. According to the rule it is usually several days before chemosis subsides sufficiently to see the cornea to its upper limit. When this stage arrived with the patient, it was a question as to what was going to be the outcome. For several days it was feared that the ulceration was progressing at the nasal end, and that more cauterization would be required. The burned area now showed green staining with fluorescein. In a few more days the ulcer began healing at its extremities, and in a few weeks entirely epithelialized over, with only a faint haze in the new-formed corneal tissue which filled out the deep trough to its normal curvature: The preceding describes the course in the right eye. When it was treated on May 29, there was no trouble with the left, and the patient was told she need not come on Decoration day, May 30. On that afternoon, however, she saw her physician, who looked at her eyes and observed nothing amiss with the left. When she came to me on the morning of May 31, the left eye had exactly the same feeling and appearances as did the right when first seen. The ulcer was touched with phenol, and notice given that if it extended the same measures would be adopted, as were used with the right, which were carried out in the same manner on June 1. This eye healed as did the first, without giving any anxiety as to its extending or the likelihood of it needing further cauterizing. The patient came daily, with a few exceptions, throughout June. During this period the cornea of each eye was anaesthetic to the touch of a cotton thread. There was very little discomfort in the eyes and the only protection was dark glasses when in bright light. While there was no sign of iritis, atropine and argyrol were put into the eyes at her daily visits, after washing out with boric solution. Tension was taken by fingers daily; I also used viosterol daily which for several years I have instilled in eyes, whose corneal nutrition it was desired to raise, but of course I would not be capable of having an opinion from such limited observation. Since beginning this practice I read mention at a Belgian society of its use under their name for it, to prevent symblepharon after burns; and some of the authors or discussers of rodent ulcer papers have also recommended it. Throughout July she came for observation at increasingly long intervals of one or two weeks, and was fully released on August 11, with perfect sensation returned to the corneae, full near vision with 
glasses, and $20 / 40$ in each eye. She had quite a large hyperopia with astigmatism, which had never been corrected, and more or less amblyopia exists almost always under this condition. She says her eyes seemed in every respect as good as any time in her life, except for presbyopia. In each eye the trough made by curetting had filled out, the corneal haze had gone, the cornea was absolutely clear, and nothing left to remind her of what had been, except a memory. In the discussions to which reference is made in the papers quoted, one of the discussers said: "If the eye recovered, then it was not rodent ulcer." Dr. Gifford negatived this by the heretofore mentioned paper in the Arch. of Ophthal. "Rodent or Mooren's Ulcer of the Cornea. Report of three cases with healing." The most notable result of the treatment used in my case, is the perfect transparency of the regenerated cornea. Regardless of the naming of the disease, I wish again to call attention to the fact that in any extensive ulceration of the cornea in which massive cyanide injections are made, the opacity of the regenerated tissue is much less than in similar conditions where the injection is not made. In a paper read by the writer before the Academy of Ophthalmology and OtoLaryngology, published in the Transactions for 1915 on p. 206 occurs the following :-

"In the graver forms of corneal ulceration, injection is the most potent means of control we possess, either alone, or in conjunction with the usual treatment employed, such as iodine, carbolic acid, actual cautery, curetting, paracentesis, or iridectomy. The clearness of scar tissue is a striking feature, as compared to what is otherwise usual. Instances have been observed where quite efficient vision was restored, where ordinarily leucoma would have precluded this possibility. In those rapidly destructive ulcerations due to suppuration of the tear-sac, it is my practice to open the - tear-sac, destroy it completely by the electro-cautery, burn the ulcer with the same, and then give an injection-all at one sitting. When done sufficiently early, the result is all that could be desired; a day or two of delay will make it too late. In one case of typical rodent ulcer of the cornea, injection, with antiseptics utterly failed. Three subsequent eyes with rodent ulcer were curetted, burned with the cautery and injected at the same sitting, and healed as promptly and smoothly as the simplest forms of ulcer. They had the ulceration extending well into the sclera, which is the characteristic feature for diagnosis. These cases are reported to bring out the advantages of adding injections to routine measures, in cases where those measures alone, and in jections alone might both fail. In the annals of Ophthalmology for July, 1915, a case is cited of a cure of an extensive retinal detachment by combined subconjunctival injection and galvano-puncture of sclera." 